\title{
Robust unidimensional PZ Summation
}

Raoni de Carvalho Costa Alves*1, DSc. Sérgio Adriano Moura Oliveira ${ }^{2},{ }^{1}$ Petróleo Brasileiro S.A., ${ }^{2}$ LENEP/UENF, ${ }^{2}$ INCT-GP and ${ }^{2}$ Invision Geophysics

Copyright 2021, SBGf - Sociedade Brasileira de Geofísica

This paper was prepared for presentation during the $17^{\text {th }}$ International Congress of the Brazilian Geophysical Society held in Rio de Janeiro, Brazil, 16-19 August 2021.

Contents of this paper were reviewed by the Technical Committee of the $17^{\text {th }}$ International Congress of the Brazilian Geophysical Society and do not necessarily represent any position of the SBGf, its officers or members. Electronic reproduction or storage of any part of this paper for commercial purposes without the written consent of the Brazilian Geophysical Society is prohibited.

\section{Abstract}

PZ summation is a mandatory step in ocean bottom sensor multi-component PP seismic processing flow, to attenuate receiver ghost and peg leg multiples from upgoing wavefield. It consists of an adaptive sum between the hydrophone and geophone components to adapt the vertical component to pressure in a least square sense. However, in spurious situations (nongaussian noised conditions), a robust method IRLS (Interactive Re-weighted Least Square) may be a better choice to run linear convolutional adapting filter. That proposal was applied on receiver gathers of the OBC data from Jubarte area, Brazil, using near receiver stack sections to quality control. Similar quality was obtained when short adaptive windows were used, but better quality was attained when applied on a selected near receiver stack section, as compared to Wiener-Levinson PZ summation results. The proposed method became a robust option to run $\mathrm{PZ}$ summation and perform the unidimensional upgoing and downgoing field decomposition, processing the first order receiver ghost and peg leg multiples.

\section{Introduction}

In petroleum production zones, multi-component seafloor seismic have been used as a successful technique to increase the fold under production facilities obstacles, improving the reservoir monitoring. However, the processing flow differs from conventional streamer data processing because the difference between source and receiver depth provides an asymmetric raypath.

Amundsen and Reitan (1995) and Soubaras (1996), published a method to process the acoustic components, the hydrophone and vertical geophone. Later Beresford and Janex (1996), Schalkwijk, Wapenaar and Verschuur (2003), Muijs, Robertsson and Holliger (2007), Wang and Grion (2008), Edme and Singh (2009), and Hugonnet et al. (2011), approached the theme of the ocean bottom seismic data processing.

Hugonnet et al. (2011), summarized the aspects of the hydrophone and geophone processing, based on Soubaras (1996), and called it as PZ summation (pressure with vertical particle velocity), generalizing it to the three-dimensional notation, under the least square sense. However, Guitton and Verschuur (2004), explain that under non-gaussian distribution, the least square method provides poor fitted results from adaptive sum, once that is highly affected by spurious measures, and suggest the robust method IRLS (SCALES; GERSZTENKORN, 1988) to solve the inversion with $L_{1}$ norm minimization. For concept aligning, robustness means the capacity to provide physical solutions under non-stationary and spurious noised conditions, avoiding high frequency oscillations in the results.

In this paper we propose to apply a linear convolutional IRLS adapting filter instead of the least square filter in the formulation of the PZ summation, to accomplish a unidimensional robust acoustic decomposition, separating the upgoing and downgoing wave fields, to treat the receiver ghost, and provide inputs to treat the peg leg multiple noise on the surveyed seafloor PP seismic data.

The adapting IRLS filter was applied to PZ summation on OBC Seismic data from Jubarte area, in Espírito Santo Basin.

\section{The Interactive Re-weighted Least Square method}

The IRLS method is based on asymptotic hyperbola $g(x)$ to approximate the $L_{1}$ norm curve $f(x)$, allowing the numerical $L_{1}$ norm differentiation once that $|x|=\sqrt{|x|^{2}+\epsilon^{2}}$ (Figure 1).

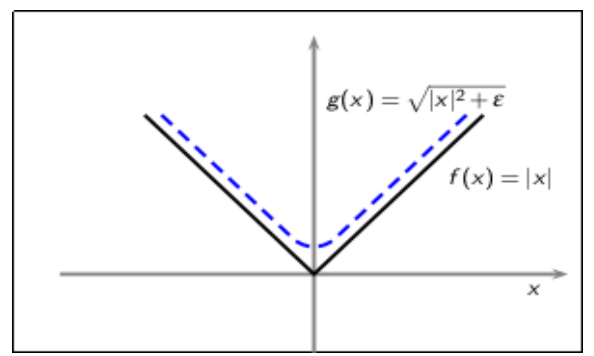

Figure 1 - Asymptotic approximation $g(x)$ which allow to differentiation of the $L_{1}$ norm curve $f(x)$.

Developing the derivative of the asymptotic hyperbola, we found the linear system below to solve iteratively the nonlinear system from L1 norm minimization

$$
\left[M^{T} A M+\mu B\right] f_{k}=M^{T} A X
$$

where $A=\operatorname{diag}\left(1 / \sqrt{\Delta^{2}+\epsilon}\right)$ is the inverse of the modeling deviation $\Delta=\left|X-M f_{k}\right| L_{1}$ norm diagonal 
matrix, and $B=\operatorname{diag}\left(1 / \sqrt{f_{k-1}^{2}+\epsilon}\right)$ is the inverse of the previous iterated filter $f_{k-1} L_{1}$ norm diagonal matrix; $k$ is the iterating index; $\epsilon$ and $\mu$ are the method parameters, which can be respectively described as the approximation accuracy controlling and the matrix regularizing to avoid division by zero (stabilizing the solution); $M$ is the model, $f_{k}$ the filter and $X$ the data concerning the adaptive sum $X-M f_{k} \approx 0$. The first iteration provides the least square solution (OLIVEIRA; LUPINACCI, 2013). To optimize it, we inserted the filter from the Wiener-Levinson algorithm (GOLUB; VAN LOAN, 1996; ROSA, 2018) as the first input to start the iterative method.

\section{The pressure with vertical particle velocity summation}

To treat the receiver ghost and peg leg in ocean bottom multi-component seismic data, Hugonnet (2011) summarized the formulation of $\mathrm{PZ}$ summation based on Soubaras (1996) by $U=H+f_{0} * G$ and $D=H-f_{0} * G$, where $U$ and $D$ are respectively upgoing and downgoing wavefields, $H$ and $G$ are hydrophone and geophone data components, and $f_{0}=\arg \min \left\|H^{\prime}-f * G^{\prime}\right\|_{2}^{2}$ is the least square filter $f_{0}=\left[G^{\prime T} G^{\prime}+\lambda I\right]^{-1} G^{T^{T}} H^{\prime}$, which may be solved by Wiener-Levinson algorithm, with the regularizing whitenoise $\lambda$.

The adaptive sum is applied due to differences between hydrophone and geophone signals, related mainly to coupling and its scalar and vector measures. In addition, because of the polarizing feature of the geophone (Figure 2 ), the notches from the receiver ghost appear on the amplitude spectra with opposite peaks and troughs on hydrophone and geophone components (Figure 3).

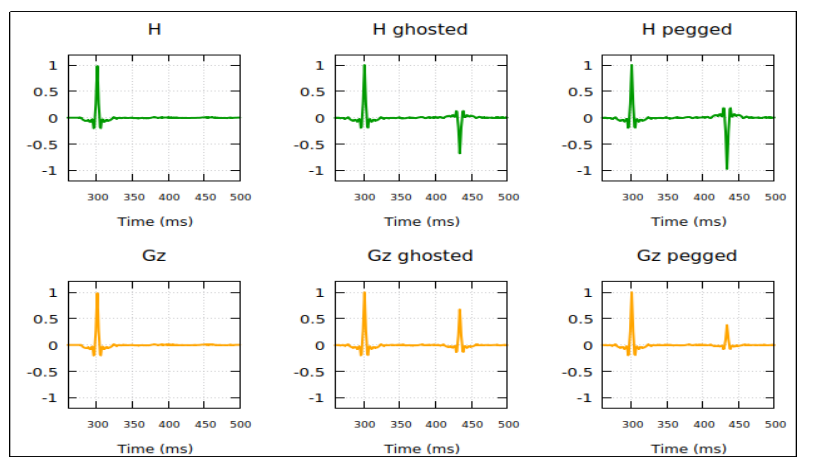

Figure 2 - Synthetic traces simulating multiple events, ghost and peg leg, to acoustic multi-component data composed by hydrophone and vertical geophone records.
To compensate that effect, Soubaras (1996) proposed the crossghosting operation preceding filter calculation, marked by $H^{\prime}=g_{0} * H$ and $G^{\prime}=h_{0} * G$, where $h_{0}$ and $g_{0}$ are respectively the deterministic first order hydrophone and geophone ghost operators, which were built in time domain considering the normal incidence angle $(\theta=0)$ and time delay as $\Delta t=2 z \cos \theta / v$ (Figure 4), as well as the polarizing feature of the geophone, the reflectivity $r$ from free air surface which is about -1 , the water column velocity $v$ by around 1500 meters per second and the geometric spreading coefficient $E$, by around 0.98 (ROSA, 2018), providing us $h_{0}=\delta_{t}-r E \delta_{t-\Delta t}$ and $g_{0}=\delta_{t}+r E \delta_{t-\Delta t}$, where $\delta_{t}$ is the delta function.

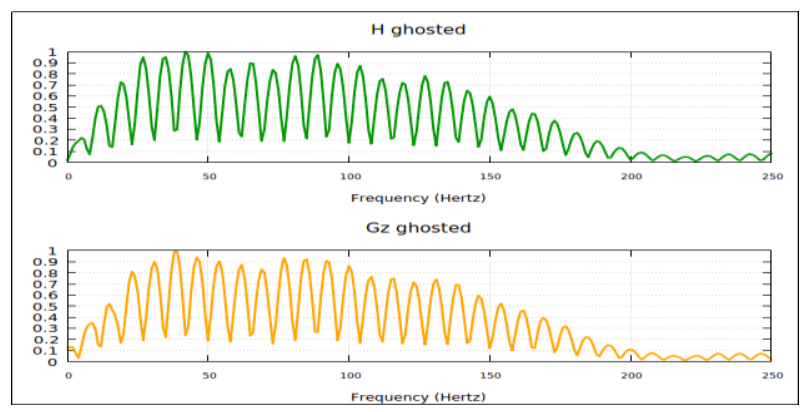

Figure 3 - Amplitude spectra for synthetic hydrophone and vertical geophone contaminated by the receiver ghost noise.

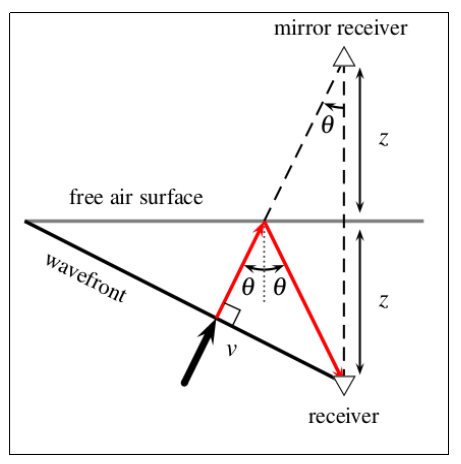

Figure 4 - Associated geometry to receiver ghost delay. Adapted from Rosa (2018).

To peg leg attenuation, we have the adaptive sum $P=U-p_{0} * D$, where $P$ is the un-pegged data, $U$ and $D$ are respectively the upgoing and downgoing wavefields from deghosting, and $p_{0}=\left[D^{T} D+\lambda I\right]^{-1} D^{T} U, \quad$ is the least square predictive filter, once the downgoing is a delayed version of the upgoing wavefield (SOUBARAS, 1996; ANG et al., 2010; EDME; SINGH, 2009; HUGONNET et al.2011), 


\section{The Robust PZ summation}

With the crossghosting operation stated, we propose a small modification on that formulation, substituting least square filter by IRLS filter, obtaining

$$
U=H+f_{k} * G ; D=H-f_{k} * G
$$

where $f_{k}=\arg \min \left\|H^{\prime}-f * G^{\prime}\right\|_{1}^{1}$ is now the IRLS filter given by $f_{0}=\left[G^{\prime T} A G^{\prime}+\mu B\right]^{-1} G^{\prime T} A H^{\prime}$, which was solved by outer product Cholesky factorization (GOLUB; VAN LOAN, 1996) because the matrix system is positive definite (LI; LI; LU, 2016). In this case, we have the modeling deviation as $\Delta=H^{\prime}-f_{k-1} * G^{\prime}$. The methods parameters $\epsilon$ and $\mu$, refer respectively to the $L_{1}$ norm accuracy approximation and to matrix regularization.

Similar formulation is developed to depegleging, so that $P=U-p_{k} * D$, where the IRLS predictive filter is $p_{k}=\left[D^{T} A D+\mu B\right]^{-1} D^{T} A U$, in this case, we have the modeling deviation as $\Delta=U-p_{k-1} * D$.

\section{Data preconditioning}

\section{Selecting}

The introduced method for PZ summation, was applied on acoustic components, hydrophone and accelerometer, of the OBC data 0364_4D_JUBARTE_PRM_MONITOR_ 01 from Jubarte area in Espírito Santo Basin, available from ANP to academic laboratory LENEP/ UENF. A receiver line, parallel and below the shot line, was selected (Figure 14), to evaluate the method on the receiver stacked line and the receiver gathers.

Before submitting the data to the robust unidimensional PZ summation, the designature, resampling and filtering steps was applied.

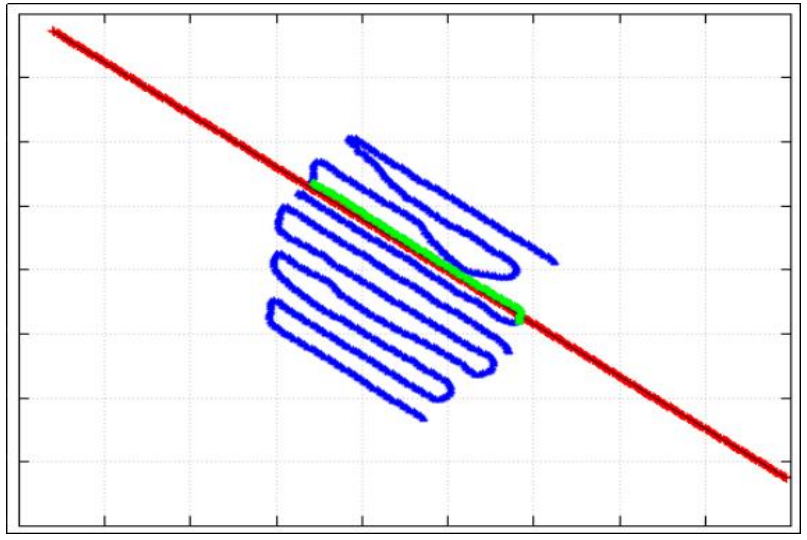

Figure 14 - Green receiver line represents selected receivers close to the red shot line, to simulate a bidimensional survey. North is up to vertical axis.

\section{Designature}

Before submitting the data to the robust PZ summation, a designature processing was applied, using a statistically derived wavelet estimated from the flattened water bottom from the receiver stacked line, to calculate the predictive filter to debubbling and a phase rotation to provide a symmetric zero phase wavelet (Figures 15, 16 and 17). After designature, the data was resampled to half of original input sample rate and submitted to anti-alias filtering.

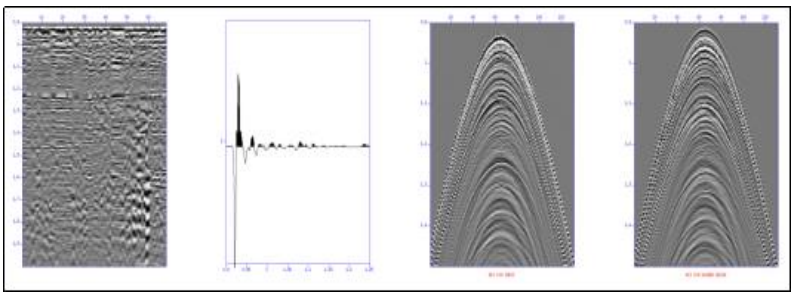

Figure 15 - Hydrophone statistical designature. From left to right: flatted stacked section; statistical wavelet; input gather; output designature gather.

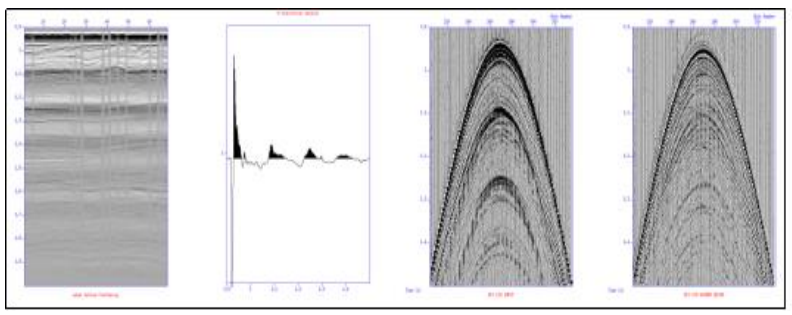

Figure 16 - Accelerometer statistical designature. From left to right: flatted stacked section; statistical wavelet; input gather; output designature gather.

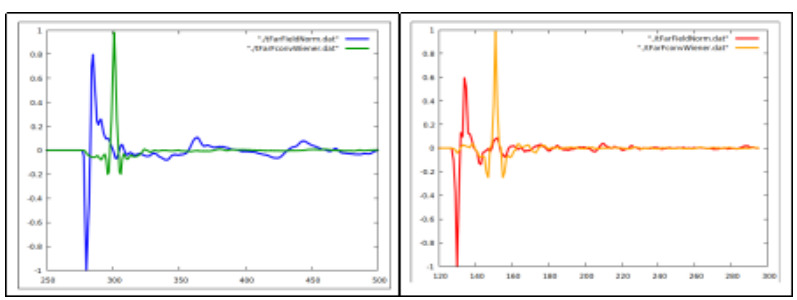

Figure 17 - Zero phase debubbled resulting wavelets from designature. From left to right: blue green hydrophone wavelet; red orange accelerometer wavelet.

\section{P Filtering and $Z$ Integration}

The hydrophone was low cut filtered (Figures 18 and 19) to attenuate anomalous low frequency band, and the accelerometer was integrated to simulate the geophone data, once the particle velocity is the derivative of the particle acceleration. 


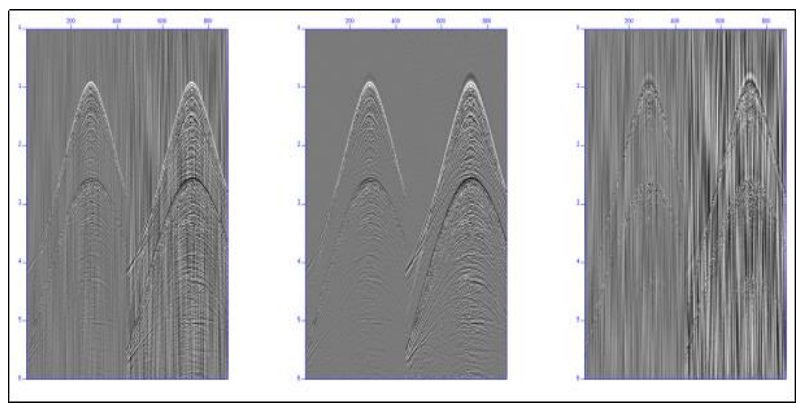

Figure 18 - Hydrophone low cut filtering, in time domain. From left to right: input, output, difference.

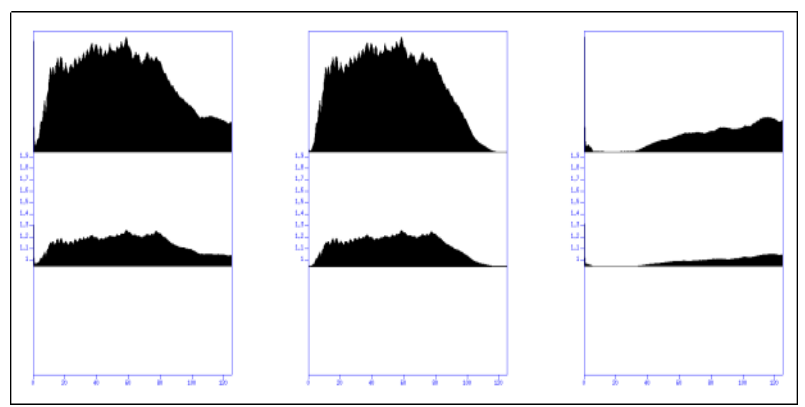

Figure 19 - Hydrophone low cut filtering, in frequency domain. From left to right: input, output, difference.

\section{Results}

\section{Deghosting}

This process is divided in two steps: filter calculation and filter application on receiver gather. During the first step, only one filter is calculated for the receiver gather, using an estimation window on near receiver stacked section. The sum runs on the stacked section to evaluate the filter quality. After the calculation, the filter is applied on all the receiver gather traces and the trace to trace sum runs before the stack. The filter quality is evaluated on both, gathers and stacked sections. We used two sizes for the estimating window, a long and a short, and the results were evaluated.

\section{Deghosting - long window}

Due to the multiple events contained in the long estimation window, the crossghosting was applied before the filter calculation. The multiple energy inserts spurious conditions violating least squares premises. The results on stacked receiver lines verify the robustness of the IRLS filtering over the Wiener-Levinson filtering (Figures 20 and 21).

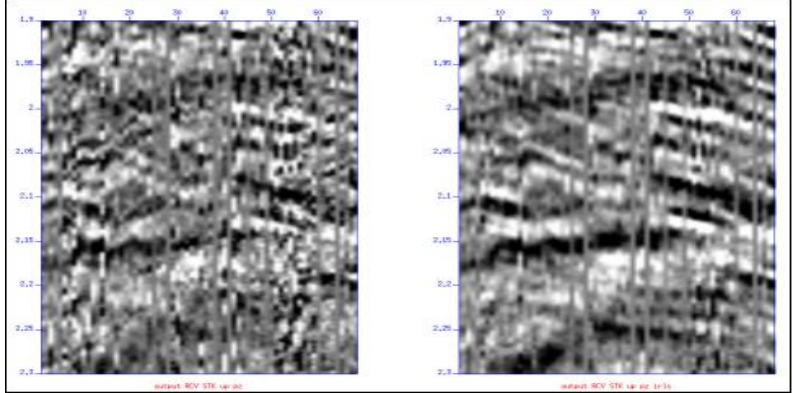

Figure 20 - Upgoing wavefields from Wiener-Levinson, left, and IRLS, right, $P Z$ summations, shown at a detailed area on the stacked receiver line section.

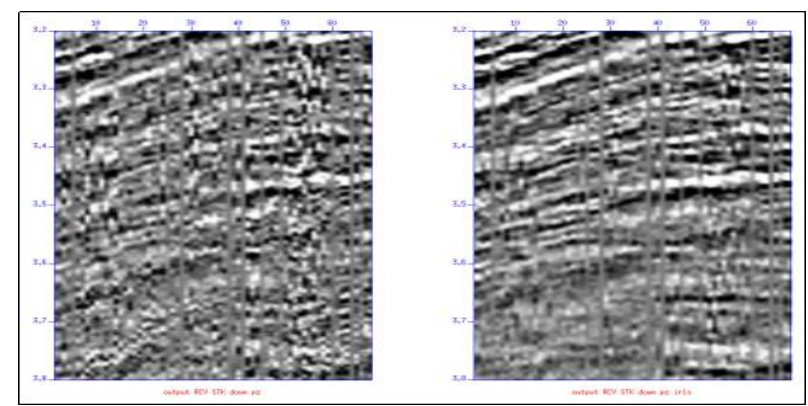

Figure 21 - Downgoing wavefields from WienerLevinson, left, and IRLS, right, $P Z$ summations, shown at a detailed area on the stacked receiver line section.

Though the promising results observed on stacked receiver lines, when applied on receiver gathers, due to unidimensional assumption used to obtain the ghost operator, modeling artifacts contaminate resulting wavefield receiver gathers, which may be compensated if we consider the real incidence angle regarding the offset trace in the receiver gather, calculating a bi-dimensional receiver ghost operator. We considered a normal incidence on horizontal water bottom reflector, so the receiver ghost operator was built only for this unidimensional case, and good results were obtained for the near offset traces in the receiver gathers, coinciding with the interception zone between the operator artifact and the real ghost event (Figure 22).

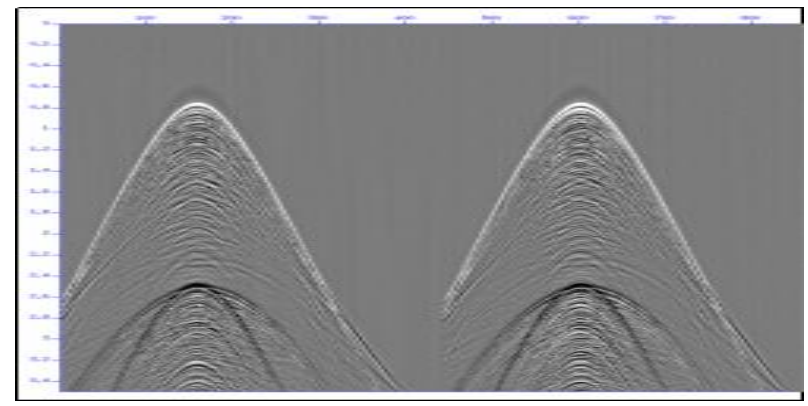

Figure 22 - IRLS downgoing wavefield receiver gather examplifying the ghost operator artifact, which was 
observed on all wavefield extracted from Wiener and IRLS unidimensional acoustic decomposition with long window.

\section{Deghosting - short window}

Because the estimating short window is between water bottom and the first order multiple, the multiple energy does not contribute to spurious conditions, and the notches does not appear on the components amplitude spectra, thus the crossghosting step was excluded from filter calculation. The results show us similar quality in the gathers and the stacked receiver lines (Figures 23 and 24), with negligible differences that enhance the amplitude spectra in useful frequency band in the IRLS upgoing field and reduce the amplitude level on high frequency band from quality control window in the IRLS downgoing wavefield, compared to Wiener-Levinson results.

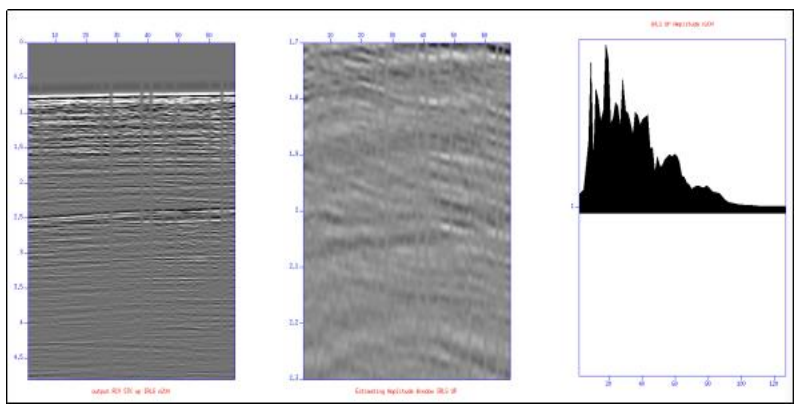

Figure 23 - IRLS upgoing stacked receiver section, left; quality control window between water surface and bottom, center; and amplitude spectrum from the one, right.

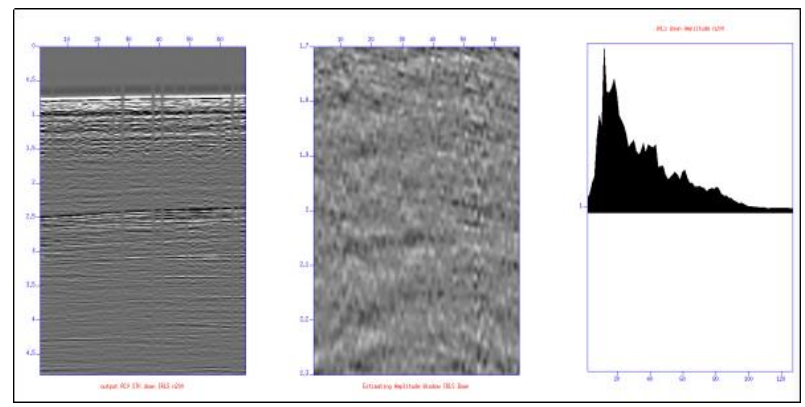

Figure 24 - IRLS downgoing stacked receiver section, left; quality control windown between water surface and bottom, center; and amplitude spectrum from the one, right.

Due to the exclusion of the multiples from inside the short estimation window, the ghost operator artifact does not appear on the resulting receiver gathers, for example we have showed the IRLS downgoing wavefield gathers in Figure 25.

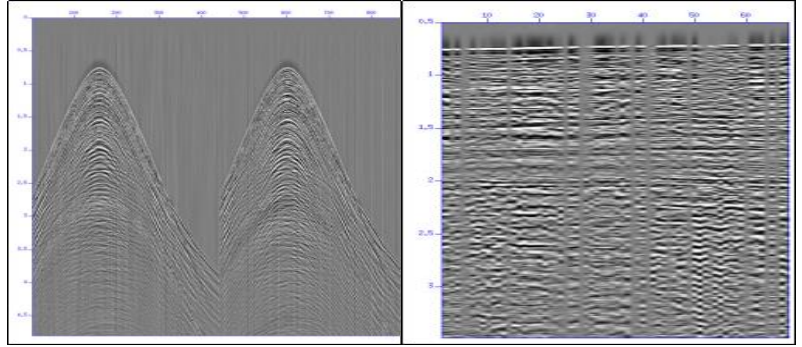

Figure 25 - IRLS downgoing wavefield receiver gather exemplifying absence of the ghost operator artifact, which was observed on all wavefield extracted from Wiener and IRLS unidimensional acoustic decomposition with short window.

\section{Depeglegging}

The depeglegging proceeding was applied with short window IRLS upgoing and downgoing wavefields as inputs. The results of applied IRLS predictive filter (Figure 26) show us similar quality in the gathers and on their corresponding receiver lines stacked, when compared to Wiener-Levinson predictive filter results.

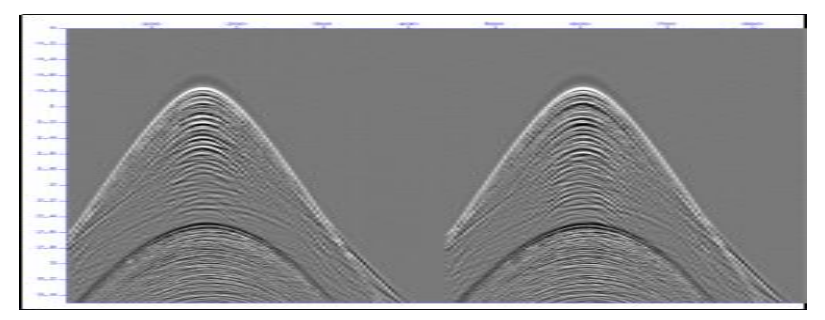

Figure 26 - IRLS depegged receiver gather, left, and stacked section from that, right. The quality was like Wiener results.

\section{Conclusions}

The IRLS showed up a robust method under spurious and least square violating situations. We recommend using that robust method when facing any of these situations. However, in situations close to the required by the least square sense, the slow results from IRLS become like the quality of the fast results from Wiener-Levinson based method, providing small improvements.

In context of 4D seismic processing, where small data quality improvements on each step are important to aggregate value to the final product, we may prefer applying robust techniques, and the IRLS method may be a good choice.

We recommend, but is not mandatory, a previous processing preceding the PZ summation, with designature and noise attenuation.

For the processing cost, the Wiener-Levinson filter is obtained by $\mathrm{n}^{2}$ float point operations, while the Cholesky IRLS filter is by $n^{3} / 3$ float point operations per iteration. 
To perform the acoustic decomposition for deghosting, we must evaluate the size of the water column, to select the correct estimation window. When the estimation window contains multiple reflections, i.e superposition between receiver ghost and peg leg, we recommend the application of the crossghosting operations before filter calculation. Thus, on shallow water bottom seismic data, it is difficult to determine an estimation window free of multiples; and on deep water bottom, the unidimensional solution for the ghost operator generates artifacts on receiver gather outputs.

The introduced method can be expanded to multidimensional case, if the correct incidence angle is calculated for each offset trace in the gather, so that the deterministic ghost operator can be correctly calculated, avoiding artifacts in outputs, as well as the filtering operation running to matrix or volumetric filter (multidimensional convolution).

\section{Acknowledgments}

We thank ANP for the session of the data used in this research. We also thank PETROBRAS and LENEP/UENF; as well as Adriano de Pinho Lima, Anderson Franco, Fernando Moraes, Marco Ceia, Jorge Nicolas Hounie and Marcella Rapinni Braga, for their contribution to the development of this work.

\section{References}

AMUNDSEN, L.; REITAN, A. Decomposition of multicomponent sea-floor data into upgoing and downgoing p- and s-waves. Geophysics, v. 60, p. 563572, março-abril 1995.

BERESFORD, G.; JANEX, G. A practical approach to obc summation and geophone calibration in areas of shallow water and hard seafloor. In: 9th International Congress of the Brazilian Geophysical Society. SBGF, Salvador, Brasil: [s.n.], 1996.

EDME, P.; SINGH, S. C. Receiver function decomposition of obc data: theory. Geophysics, v. 177, p. 966-977, fevereiro 2009.

GOLUB, G. H.; VAN LOAN, C. F. Matrix Computation. 3a. ed. Johns Hopkins: Londres, 1996.

GUITTON, A.; VERSCHUUR, D. J. Adaptive subtraction of multiples using the 11-norm. Geophysical Prospecting, EAGE, v. 52, p. 27-38, 2004.

HUGONNET, P. et al. Pz summation of $3 d$ waz obs receiver gathers. In: 73rd EAGE conference \& Exhibition incorporating SPE EUROPEC. Viena, Austria: [s.n.], 2011.

LI, Z.-X.; LI, Z.-C.; LU, W. Multichannel predictive deconvolution based on the fast iteractive shrinkagethresholding algorithm. Geophysics, SEG, v. 81, n. 1, p. 17-30, janeiro 2016.

MUIJS, R.; ROBERTSSON, J. O. A.; HOLLIGER, K. Data-driven adaptive decomposition of multicomponente seabed seismic recordings: Application to shallow-water data from the north sea. Geophysics, v. 72, n. 6, p. V133V142, novembro-dezembro 2007.

OLIVEIRA, S. A. M.; LUPINACCI, W. M. L1 norm inversion method for deconvolution in attenuating media. Geophysical Prospecting, EAGE, p. 1-7, 2013.

ROSA, A. R. Análise do Sinal Sísmico. 2a. ed. SBGf: Rio de Janeiro, 2018.

SCALES, J. A.; GERSZTENKORN, A. Robust methods in inverse theory. Inverse Problems, UK, n. 4, p. 10711091, março 1988.

SCHALKWIJK, K. M.; WAPENAAR, C. P. A.; VERSCHUUR, D. J. Adaptive decomposition of multicomponent ocean-bottom seismic data into downgoing and upgoing p- and s-waves. Geophysics, v. 68 , p. 1091-1102, maio-junho 2003.

SOUBARAS, R. Ocean bottom hydrophone and geophone processing. In: 66th Annual International Meeting. SEG: [s.n.], 1996.

WANG, Y.; GRION, S. Pz calibration in shallow waters: the britannia obs example. In: Las Vegas Annual Meeting. SEG: [s.n.], 2008. p. 1088-1092. 